Clinical trial

\title{
Pregnancy outcomes in Portuguese women with multiple sclerosis: The PREGNIMS study
}

\author{
Ana Novo $^{\mathrm{a}}$, Juliana Castelo ${ }^{\mathrm{b}}$, Ary de Sousa ${ }^{\mathrm{c}}$, Isabel Amorim ${ }^{\mathrm{d}}$, José Nuno Alves ${ }^{\mathrm{e}}$, \\ Margarida Calejo ${ }^{f}$, Ana Monteiro ${ }^{g}$, Marta Arenga ${ }^{h}$, Motasem Shamasna ${ }^{i}$, José Vale $^{j}$, \\ Maria José Sá ${ }^{g, k}$, Filipe Palavra, ${ }^{1, m}$,* \\ ${ }^{a}$ Neurology Department, Centro Hospitalar e Universitário de Coimbra, Coimbra, Portuga \\ ${ }^{\mathrm{b}}$ Neurology Department, Centro Hospitalar de Entre o Douro e Vouga, Santa Maria da Feira, Portugal \\ ${ }^{\mathrm{c}}$ Neurology Department, Centro Hospitalar de Lisboa Central, Lisboa, Portugal \\ ${ }^{\mathrm{d}}$ Neurology Department, Centro Hospitalar de Lisboa Norte, Lisboa, Portugal \\ ${ }^{\mathrm{e}}$ Neurology Department, Hospital de Braga, Braga, Portugal \\ ${ }^{\mathrm{f}}$ Neurology Department, Centro Hospitalar e Universitário do Porto, Porto, Portugal \\ ${ }^{g}$ Neurology Department, Centro Hospitalar e Universitário de S. João, Porto, Portugal \\ ${ }^{\mathrm{h}}$ Neurology Department, Centro Hospitalar da Cova da Beira, Covilhã, Portugal \\ ${ }^{\mathrm{i}}$ Neurology Department, Centro Hospitalar e Universitário do Algarve, Faro, Portugal \\ ${ }^{\mathrm{j}}$ Neurology Department, Hospital Beatriz Ângelo, Loures, Portugal \\ ${ }^{\mathrm{k}}$ FP-ENAS (UFP Energy, Environment and Health Research Unit), Faculty of Health Sciences, Fernando Pessoa University, Porto, Portugal \\ ${ }^{1}$ Centre for Child Development, Neuropediatrics Unit, Hospital Pediátrico, Centro Hospitalar e Universitário de Coimbra, Avenida Afonso Romão, 3000-602 Coimbra, \\ Coimbra, Portugal \\ ${ }^{\mathrm{m}}$ Laboratory of Pharmacology \& Experimental Therapeutics, Coimbra Institute for Clinical and Biomedical Research (iCBR), Faculty of Medicine, University of Coimbra, \\ Coimbra, Portugal
}

\section{A R T I C L E I N F O}

\section{Keywords:}

Multiple sclerosis

Pregnancy

Newborn

\begin{abstract}
A B S T R A C T
Introduction: Several questions about pregnancy in women with multiple sclerosis (MS) have been discussed, but clarification is still needed in some very practical issues. Portuguese data on this subject remain scattered and need to be analyzed in order to standardize clinical practice.

Objective: This study aimed to describe and analyze the impact of MS on pregnancy and perinatal health of children born to Portuguese mothers with the disease.

Material and methods: This is a multicenter, retrospective study of a cohort of Portuguese women with MS who were pregnant and who gave birth between 01/01/2011 and 31/12/2015. Demographic and clinical data related to maternal disease, pregnancy progression and events, childbirth and newborn health were collected. Results: Ninety-seven women were recruited and 90 live births were evaluated. The mean maternal age at conception was 32.5 years, and $63.9 \%$ had no relapses in the previous year $(98.0 \%$ had a relapsing-remitting MS and the EDSS score was $\leq 3$ in $92.8 \%$ of the cases). Only $50.5 \%$ of the women had a preconception specific evaluation and 60 children were exposed to immunomodulatory therapies during pregnancy. Nineteen women had relapses during pregnancy. Childbirth was induced in $22.7 \%$ of the cases, and the caesarean section rate was $34 \%$. Children exposed to immunomodulatory drugs during pregnancy had a lower birth length $(p=0.014)$, and there was also a trend toward lower birth weight $(p=0.054)$ in these newborns. Pre-conception EDSS score negatively correlated with the duration of pregnancy $(r=-0.22 ; p=0.029)$, weight $(r=-0.23 ; p=0.031)$ and cephalic perimeter at birth $(r=-0.24 ; p=0.033)$. There was no relationship between the occurrence of relapses or progression in EDSS score during pregnancy with any variables related to the newborn.

Conclusions: In our cohort, it has been confirmed that MS has no negative effect on pregnancy or on children's perinatal health. However, the use of immunomodulatory drugs may have some impact on newborns' somatometric features.
\end{abstract}

\footnotetext{
* Corresponding author.

E-mail address: fpalavra@fmed.uc.pt (F. Palavra).
} 


\section{Introduction}

Multiple sclerosis (MS) usually affects women in childbearing age, making the effect of pregnancy on the disease (and vice versa) an important clinical issue (Voskuhl and Momtazee, 2017). It is now well established that the risk of relapse significantly declines during pregnancy, particularly during the third trimester, only to increase threefold in the first three-to-four months postpartum, compared with the pre-pregnancy period (Nelson et al., 1988; Confavreux et al., 1998). This $70 \%$ reduction in disease activity during the third trimester was firstly reported in the PRIMS (Pregnancy in Multiple Sclerosis) study, a relatively large-scale multicenter prospective study (Confavreux et al., 1998). Nevertheless, inconsistent results have been observed regarding the effect of MS on pregnancy outcomes and on the prognosis for longterm disability. While the majority of studies claims that MS does not increase adverse pregnancy outcomes (Poser and Poser, 1983; Sadovnick et al., 1994; Mueller et al., 2002; Van der Kop et al., 2011; Finkelsztejn et al., 2011), some studies reported that mothers with MS are more likely to have infants with low birth weight for the gestational age (Kelly et al., 2009; Chen et al., 2009; Dahl et al., 2005, 2008; Hellwig et al., 2008), or prematurely born (Chen et al., 2009), compared to healthy women. Other studies reported a greater likelihood of assisted vaginal delivery (Jalkanen et al., 2010) and cesarean delivery (Kelly et al., 2009) for those women diagnosed with MS. Regarding long-term disability, no studies have found a harmful effect of pregnancy. While some studies found no association (Ramagopalan et al., 2012; Thompson et al., 1986; Weinshenker et al., 1989), others suggested a protective effect (Jokubaitis et al., 2016; Runmarker and Andersen, 1995; D'Hooghe et al., 2012, 2010) on this disorder. Moreover, the role of breastfeeding as a modifiable risk factor of postpartum relapses remains unclear: previous studies found either no association (Nelson et al., 1988; Confavreux et al., 1998; Portaccio et al., 2011) or a protective effect, particularly of exclusive breastfeeding (Langer-Gould et al., 2009; Gulick, 2002).

Over the last few years, some studies addressed the effect of diseasemodifying treatments (DMTs) for MS on pregnancy and birth outcomes and on disease course. There is some evidence that pregnant women exposed to DMTs during pregnancy or in the 2 years preceding conception are less likely to have postpartum relapses (Fragoso et al., 2013; Hughes et al., 2014) and EDSS progression (Fragoso et al., 2013) than women who were not exposed to any DMT. Most treating physicians tend to advice the resumption of DMT after delivery, although the optimal time to restart the DMT and other appropriate measures to prevent postpartum relapses remain unclear (Alroughani et al., 2018).

A recent Portuguese unicentric study also concluded that MS did not have a deleterious effect on pregnancy outcome (Jesus-Ribeiro et al., 2017). However, there is still a need for collecting multicentric data as an initial effort to create a Portuguese MS Pregnancy Registry. This multicentric study aims to describe and analyze the effect of MS on pregnancy and perinatal health of children born to mothers with this disease in Portugal.

\section{Methods}

"Pregnancy outcomes in Portuguese women with multiple sclerosis" (PREGNIMS) is an investigator-initiated, multicenter, retrospective cohort study conducted to describe the effects of MS on pregnancy and fetal outcomes in Portuguese women with MS. Recruitment was consecutively performed, including female patients with a previous diagnosis of MS fulfilling 2010 McDonald Criteria (Polman et al., 2011), who had at least one delivery between 1st January 2011 and 31st December 2015, and that were under follow-up in specialized MS clinics in nine Portuguese hospitals. Women with other neurological conditions, deemed to impact pregnancy outcomes, were excluded. We collected data concerning maternal clinical characteristics, pregnancy course and outcome, delivery and newborn clinical status. We extended data collection during the 1-year postpartum period to include information related to the resumption of immunomodulatory therapy (and the variables that conditioned it), the relapses, and the progression of disability during that period of time. Data were collected from medical records, individual pregnancy records, infantile individual health records and when necessary, through individual interviews with the patients. The National Data Protection Commission and the local Ethics Committees from the involved centers approved this study. Patients gave written informed consent to participate in the study.

Considering the statistical analysis, continuous variables were tested for normality using the Kolmogorov-Smirnov goodness of fit test. Baseline features are presented as frequency (percentage) for dichotomous variables, median \pm interquartile range (IQR) for quantitative variables without normal distribution and mean \pm standard deviation (SD) for normally distributed quantitative variables. Comparisons between two groups were made using independent-samples T-test for normally distributed variables, Mann-Whitney $U$ test for quantitative variables not normally distributed and Chi-square $(\chi 2)$ test for categorical variables. For matched samples the Wilcoxon test was used. Pearson's correlation (or Spearman correlation if normality was not assumed) was used to assess the correlation between quantitative clinical variables. To compare the subgroups of DMTs in relation to the somatometric characteristics of newborns ANOVA test and Bonferroni correction were applied. The level of statistical significance was set at $p<0.05$ for all analyses. IBM SPSS Statistics ${ }^{\circledR}$ (version 25) was used for statistical analysis.

\section{Results}

\subsection{MS and pregnancies}

We identified a total of 107 pregnancies in the study period. In cases corresponding to several pregnancies of the same woman, only the first gestation was included. Thus, 97 pregnancies resulting in 90 live births were eligible for analysis.

The baseline characteristics of maternal MS are resumed in Table 1. The mean maternal age at conception was $32.5( \pm 4.3)$ years (range $17-41)$. The majority of women $(n=62 ; 63.9 \%)$ had no relapses in the year prior to conception, 28 had 1 relapse (28.9\%), 6 had 2 relapses $(6.2 \%)$ and 1 had 3 relapses $(1.0 \%)$ in the same time period. Also the majority had an Expanded Disability Status Scale (EDSS) score $\leq 3$. Only $32(33.0 \%)$ women had a planned pregnancy and previously discontinued the DMT, although $49(50.5 \%)$ reported a prior consultation on family planning. In 25 cases (25.8\%), patients and/or their neurologists opted to use DMT during pregnancy, especially at very early stages, until the pregnancy was effectively diagnosed.

At the time of pregnancy diagnosis (mean $5.9 \pm 3.5$ weeks), 57 woman $(89.1 \%)$ suspended DMT. In total, 65 were exposed to DMT, mostly during the first trimester and until the pregnancy was diagnosed. The mean total exposure time was $8.0 \pm 9.2$ (range 1-41)

Table 1

Baseline characteristics of mothers' MS

\begin{tabular}{ll}
\hline Baseline characteristics & $\mathrm{n}=97$ \\
\hline Age at MS diagnosis, mean \pm SD (min-max), years & $25.6 \pm 5.6(9-36)$ \\
MS course & $95(98.0)$ \\
$\quad$ Relapsing-remitting MS, n (\%) & $1(1.0)$ \\
Primary progressive MS, n (\%) & $1(1.0)$ \\
Secondary progressive MS, n (\%) & $0.4 \pm 0.7$ \\
Annualized relapse rate prior to conception, mean \pm SD & $6.9 \pm 5.2(1-26)$ \\
Duration of MS at conception, mean \pm SD (max-min), years & $1.0 \pm 2.0(0-6)$ \\
EDSS at conception ${ }^{\text {a }}$, median \pm IQR (min-max) & $90(92.8)$ \\
EDSS $\leq 3, \mathrm{n}(\%)$ & $7(7.2)$ \\
EDSS $>3, \mathrm{n}(\%)$ &
\end{tabular}

a Measured at the last visit before pregnancy diagnosis or at the first appointment after becoming pregnant 
Table 2

Baseline characteristics of pregnancies and comparative analysis with exposure to DMT

\begin{tabular}{|c|c|c|c|}
\hline Baseline characteristics & With DMT $^{\mathrm{a}}(\mathrm{n}=65)$ & Without DMT $(\mathrm{n}=32)$ & p-value \\
\hline Result of pregnancy & & & $>0,05$ \\
\hline Live births, $\mathrm{n}(\%)$ & $60(92.3)$ & $30(93.8)$ & \\
\hline Spontaneous abortion, $\mathrm{n}$ (mean \pm SD weeks) & $4(9.3 \pm 4.7)$ & $1(8)$ & \\
\hline \multicolumn{4}{|l|}{ Pregnancy termination, n (mean, week) } \\
\hline Voluntary & $1(8)$ & 0 & \\
\hline Due to major fetal malformation & 0 & $1(11)$ & \\
\hline Pregnancy duration, mean \pm SD (min-max), weeks & $36.2 \pm 8.2(5-42)$ & $36.9 \pm 7.3(8-41)$ & $>0,05$ \\
\hline Relapses during pregnancy, n (\%) & $14(21.5)$ & $5(15.6)$ & $>0,05$ \\
\hline 1 relapse, $\mathrm{n}(1 \mathrm{~T}, 2 \mathrm{~T}, 3 \mathrm{~T})^{\mathrm{b}}$ & $8(2,4,2)$ & $5(2,3,0)$ & \\
\hline 2 relapses, $\mathrm{n}(1 \mathrm{~T}, 2 \mathrm{~T}, 3 \mathrm{~T})^{\mathrm{b}}$ & $3(1,4,1)$ & $0(0,0,0)$ & \\
\hline$\geq 3$ relapses, $\mathrm{n}(1 \mathrm{~T}, 2 \mathrm{~T}, 3 \mathrm{~T})^{\mathrm{b}}$ & $3(2,3,6)$ & $0(0,0,0)$ & \\
\hline Other gestational complications, $\mathrm{n}(\%)$ & $14(21.5)$ & $3(9.4)$ & \\
\hline Gestational diabetes & 6 & 0 & $>0,05$ \\
\hline Eclampsia & 0 & 0 & \\
\hline Preeclampsia & 5 & 1 & $>0,05$ \\
\hline Other $^{c}$ & 3 & 2 & \\
\hline
\end{tabular}

a Exposure ranging from 1 to 41 weeks;

b $1 \mathrm{~T}$ - first trimester; $2 \mathrm{~T}$ - second trimester; $3 \mathrm{~T}$ - third trimester;

c placental abruption, repeated urinary tract infections, arterial hypertension

weeks. Fifty-nine (90.8\%) patients were exposed to first-line DMT (mean exposure time $7.8 \pm 9.4$ weeks) and $3(4.6 \%)$ were exposed to second-line DMT, namely to natalizumab (mean exposure time $10.7 \pm 6.1$ weeks).

Clinical data of pregnancies are shown in Table 2, divided according to DMT exposure status. Four women intentionally maintained DMT (glatiramer acetate, GA) throughout pregnancy due to severe disease in the past and fear of disease reactivation during pregnancy or immediately after delivery.

Five women restarted treatment between 16 and 3 weeks before delivery, due to the occurrence of relapses (natalizumab was prescribed) or active disease prior to conception despite the absence of relapses throughout pregnancy [GA or intravenous immunoglobulin (IVIg)]. Table 3 depicts other immunomodulators used during pregnancy.

The relapse rate during pregnancy had a mean of 0.3 , without clear predominance in a specific trimester. There is very weak positive correlation $(r=0.224 ; p=0.027)$ between the relapse rate during pregnancy and EDSS score prior to pregnancy. Other indicators of disease severity, such as the occurrence of relapses in the 12 months prior to gestation or the duration of the disease did not influence relapse rate during pregnancy. There was also no statistically significant difference in the relapse rate between those exposed and not exposed to DMT during pregnancy, regardless of the exposure time and the DMT class (1st or 2nd line therapies).

Labor induction was necessary in $20(22.7 \%)$ cases, and epidural anesthesia was used in 71 (78.9\%) women. The different types of delivery were registered as follows: vaginal delivery $(n=38 ; 39.2 \%)$, emergent caesarean $(n=16 ; 16.5 \%)$, elective caesarean $(n=17$; $17.5 \%)$, vacuum extraction $(n=11 ; 11.4 \%)$ and forceps delivery ( $n=7 ; 7.2 \%$ ); data were missing in 8 cases $(8.2 \%)$. The main reasons for caesarean section were fetal distress $(n=15)$ and maternal disease

Table 3

Other treatments during pregnancy

\begin{tabular}{ll}
\hline Treatment characteristics & \\
\hline Corticosteroid, $\mathbf{n}(\%)$ & $12(12.4)$ \\
Total days, mean \pm SD (min-max) & $7.7 \pm 4.9(3-20)$ \\
Total dose, mean \pm SD (min-max), g & $7.5 \pm 5.0(1.5-20)$ \\
IVIg, n (\%) & $4(4.1)$ \\
Total days, mean \pm SD (min-max) & $5.8 \pm 2.9(2-8)$ \\
Total dose, median (min-max), g & $117.5(48-1280)$ \\
\hline
\end{tabular}

$(n=9)$. The need for labor induction was associated with older age at pregnancy onset $(t(37.7)=-2,23 ; p=0.031)$ and was not associated with disease clinical phenotype.

Women with relapses during pregnancy did not have higher frequency of caesarean delivery $(p=0.322)$. Conversely, the need for assisted delivery (forceps and vacuum extraction) was associated with relapses in pregnancy $\left(\chi^{2}(1)=12.4 ; p=0.001\right)$, as well as increased EDSS score between pre- and postpartum $(t(20.24)=-2.64$, $p=0.016$ ), but was not related to corticosteroid (CT) use during pregnancy $(p=0.416)$ or to somatometric characteristics of the newborn (cephalic perimeter: $t(79)=0.96, p=0.340$; birth length: $t$ (84) $=-1.37, p=0.172$; birth weight: $t(58.6)=-1.03, p=0.307)$.

In the first year after childbirth, $92.2 \%$ of women restarted DMT, but only $13.3 \%$ restarted treatment in the immediate postpartum period (during the first seven days after delivery), in accordance with their physicians' recommendation. Eighty percent chose to breastfeed and, hence, the mean delay time until DMT restart was 3.0 months. Twentyeight women $(30.8 \%)$ had at least one relapse in the first year after delivery and the mean time until this happened was 4.0 months (range 2-12). Using survival analysis, it was possible to see that the use of DMT, the type of DMT [GA, interferon beta (IFN), or second-line DMT], corticosteroids or IVIg use in pregnancy, as well as the relapse rate during pregnancy and in the prior year, did not have any impact in time until the first postpartum relapse.

The EDSS score after delivery was assessed at the first consultation with the assistant neurologist, sometimes several months after delivery, not controlling the possibility of a new relapse occurring in that interval. The median postpartum EDSS was 1.0, corresponding to a statistical significant increase in pre- and post-pregnancy EDSS score by $27.8 \%(r=0.850 ; p<0.001)$.

\subsection{MS and newborns}

The characteristics of the population of newborns are summarized in Table 4. There were no perinatal deaths and there were minor malformations in 3 babies (3.3\%): pilonidal sinus, bilateral ectasia of kidney pelvis and congenital torticollis (none of these situations required additional hospitalization). Pregnancy duration was not significantly influenced by the occurrence of relapses or by the exposure to MS treatments. On the other hand, pre-conception EDSS score negatively correlated with the duration of pregnancy $(r=-0.22$; $p=0.029)$, weight $(r=-0.23 ; p=0.031)$ and cephalic perimeter at birth $(r=-0.24 ; p=0.033)$. The occurrence of relapses or an increase 
Table 4

Characteristics of newborns

\begin{tabular}{|c|c|c|c|c|}
\hline Baseline characteristics & Total & With DMT ${ }^{\mathrm{a}}$ & Without DMT & P-value \\
\hline Weight at birth, mean $\pm \mathrm{SD}, \mathrm{g}$ & $3053.3 \pm 466.7$ & $2991.0 \pm 457.3$ & $3177.7 \pm 357.2$ & 0.054 \\
\hline Length at birth, mean $\pm \mathrm{SD}$, cm & $48.2 \pm 2.8$ & $47.7 \pm 3.1$ & $49.1 \pm 1.9$ & 0.014 \\
\hline Cephalic perimeter at birth, mean $\pm \mathrm{SD}$, cm & $34.3 \pm 1.7$ & $34.1 \pm 1.9$ & $34.7 \pm 1.3$ & 0.118 \\
\hline Prematurity, n (\%) & $5(8.3)$ & $5(8.3)$ & $0(0.0)$ & 0.266 \\
\hline $32-36$ weeks, $\mathrm{n}$ & 4 & 4 & 0 & \\
\hline 28 weeks, $\mathrm{n}$ & 1 & 1 & 0 & \\
\hline Hospitalization after birth, mean $\pm \mathrm{SD}$, days & $3.7 \pm 1.8$ & $3.9 \pm 2.1$ & $3.1 \pm 0.9$ & 0.048 \\
\hline
\end{tabular}

a Exposure ranging from 1 to 41 weeks.

of EDSS score during pregnancy had no impact on neonate weight, length and cephalic perimeter at birth.

DMT exposure at any time during pregnancy had a statistical significant impact on birth length $(t(85)=2.13 ; p=0.014)$, but not in other variables. Nevertheless, a trend toward a statistically significant difference was observed in birth weight, with those exposed to DMT tending to weight less than those not exposed $(p=0.054)$. In the subgroup analysis of DMTs there were no significant differences on newborn somatometric characteristics when comparing those exposed to first-line DMTs (GA vs IFN), GA and any other DMTs (including secondline drugs), or those exposed to GA vs no DMTs.

Children born to mothers who received DMT during pregnancy had longer hospitalizations after birth, comparing to children not exposed to DMT $(t(88)=2.00 ; p=0.048)$, but no significant medical intercurrences were documented.

\section{Discussion}

This study represents a first look into the overall reality of pregnancy outcomes in Portuguese MS patients. We were able to collect data from centers from every region of the continental territory (north, center, south) as well as from almost all major neurological centers treating MS patients, allowing a comprehensive survey of disease management practices among Portuguese neurologists and obstetricians dealing with this particular patient group.

Overall, the outcome of the patients and their offspring was good and comparable to data from other MS populations. Five percent of the pregnancies ended in miscarriage, which is in line with the findings of the PRIMS study (Confavreux et al., 1998). Other main complications included gestational diabetes (6\%) and preeclampsia (6\%), which were within the observed in other cohorts that report between $3.9 \%$ and $14.8 \%$ incidence of gestational diabetes (Van der Kop et al., 2011; Jalkanen et al., 2010) and between $4.9 \%$ and $10.7 \%$ of hypertensionrelated complications. (Kelly et al., 2009; Jalkanen et al., 2010) There were no perinatal deaths and malformations were minor in only 3 babies $(3.3 \%)$.

Differently to what is described in the literature (Nelson et al., 1988; Confavreux et al., 1998; Hellwig et al., 2012), there was a small but not statistically-significant relapse rate reduction between the pre-conception and gestational period. This is probably due to an already low preconception relapse rate in our cohort when compared to other series, which report pre-conception annualized relapse rates between 0.68-1.2 (Confavreux et al., 1998; Jalkanen et al., 2010; Hellwig et al., 2012; Fragoso et al., 2013). This could be explained by better treatment responses and/or to the fact that only women with more benign or bettercontrolled disease decided to proceed with pregnancy in our specific cultural setting. The use of, type or time of exposure to DMT during pregnancy did not seem to influence the rate of relapses during pregnancy either. Another study addressing this issue found that both patients with or without DMT use during pregnancy experienced a reduction of relapse rate throughout gestational period and that DMT use did not confer special protection (Hellwig et al., 2012).

In our cohort, we observed a significant increase in the EDSS score between the pre-pregnancy year and the post-partum period. Our finding is not in line with previous evidence showing no apparent acceleration of the progression of disability in the post-partum period compared to the earlier periods, despite the increase in the relapse rate (Confavreux et al., 1998; Ramagopalan et al., 2012; Thompson et al., 1986; Weinshenker et al., 1989). However, since we do not have data on previous evolution of each patient, it is not possible to exclude that the EDSS score might represent the natural evolution of the disease. Moreover, we cannot exclude the existence of a certain overvaluation of symptoms during the gestational period, in which there is a natural hyper-vigilance of the woman herself.

When considering the effects of MS in child's outcome, we found a weak correlation between higher pre-conception EDSS and shorter pregnancy duration, lower birth weight and lower head circumference. This probably reflects the impact of a more severe maternal MS on the pregnancy. Literature results are mixed, with some studies finding similar results (Kelly et al., 2009; Chen et al., 2009; Dahl et al., 2005, 2008; Hellwig et al., 2008) and others reporting no adverse outcomes associated with MS (Poser and Poser, 1983; Sadovnick et al., 1994; Mueller et al., 2002; Van der Kop et al., 2011; Finkelsztejn et al., 2011). The use of DMT, particularly interferon beta during pregnancy has also been associated with lower weight and length and lower gestational age at birth. (Lu et al., 2012) In our cohort, pregnancies exposed to DMT led to neonates with lower birth weight and length and this included all the preterm births; however only newborn's length was significantly different between the exposed and non-exposed pregnancies. There was also no significant association when testing for different DMTs. As such, our results point toward a relative safety of these drugs on newborn outcomes, while disease severity might have more impact on these measures.

A few studies have found that DMT use during pregnancy (mostly only during the first trimester) had a significant effect in reducing the relapse rate and disability progression postpartum. (Hellwig et al., 2012; Fragoso et al., 2013) In our cohort, we found that neither use nor duration of exposure to DMT during pregnancy appeared to have a significant impact on the time until the first postpartum relapse. We did find an increased risk of postpartum relapse in patients using two DMTs during pregnancy, most likely reflecting a more severe and active disease, requiring more intensive treatment. Since randomized trials with DMTs in pregnant women are not feasible, data from observational and open studies are a crucial source of information on this issue. Our study points toward a small risk associated with DMT use in pregnancy, albeit with no measurable benefit.

There is no consensus approach on DMT usage in a prospective mother with MS. A recent survey of European MS neurologists showed a near 50/50 divide between those who favored discontinuation of DMT previous to conception in stable relapsing-remitting MS patients and those who did not (Fernández et al., 2018). In our cohort, we found that, in around one third of cases, women and/or physicians opted to maintain DMT while attempting to conceive.

In our study, a caesarean section was performed in $34 \%$ of deliveries, which is quite comparable to data from the Portuguese general population from the same time frame of this study (2009-2015), which 
was 33.1-36.6\% (Ayres-De-Campos et al., 2015). While some studies show MS is not associated with an increased rate of caesarean sections (Van der Kop et al., 2011; Jalkanen et al., 2010), these were studies made in populations with low rates of this procedure (from $10-18 \%$ of deliveries). On the other hand, another study in a population with rates of caesarean sections closer to ours found MS to be associated with an increased risk of caesarean section.

A few studies have previously showed an increased rate of assisted delivery in patients with MS, when compared with the general population. (Dahl et al., 2005; Jalkanen et al., 2010) Results from our cohort show that more relapses during pregnancy are associated with the need for assisted delivery (forceps or vacuum extraction) and with an increase in the EDSS score postpartum. Neuromuscular weakness and/or sensitive deficits induced by relapses and fatigue due to higher disease activity might explain this increased risk. We did not find an association between assisted delivery and newborn somatometric variables, in opposition to data from the general population. (Rabei et al., 2017)

There are some limitations to this study to be considered. Firstly, its retrospective design and the possibility of recall bias in reporting data. However, the consultation of the medical records and the individual pregnancy and infantile health records reduced a possible negative effect of these circumstances in our results. Secondly, the patients presented relatively mild disability and most of them had a relapsing-remitting subtype of MS. Therefore, we could not define if the results of the current study are fully applicable to all MS patients. Finally, the lack of a control group, precluding comparisons between pregnant women with and without MS, is also a point worth mentioning.

\section{Conclusion}

Our study is the first multicenter report about the reality of pregnancy outcomes in Portuguese MS patients. Overall, our results demonstrate that MS does not appear to have a negative impact on the main outcomes of pregnancy, but a more severe maternal disease previously and during the pregnancy (EDSS score) seems to have a negative impact on the length of newborns, also conditioning a trend to lower body weight at birth. We also observed a small risk associated with DMT use in pregnancy, albeit with no measurable benefit. Due to great variability around the many features of pregnancy management in MS patients, as well as the paucity of data about newborns' outcomes, there is a need to broaden the discussion of these aspects in order to standardize clinical procedures.

\section{Acknowledgments}

This research project was part of the program "EMIN3 - Esclerose Múltipla Para Internos de Neurologia 3", which was supported by the Portuguese Group for the Study of Multiple Sclerosis (GEEM) and Biogen. Authors also acknowledge the contribution of all the coordinators of Multiple Sclerosis Clinics of the hospitals that participated in the study, by their help in collecting data.

\section{References}

Voskuhl, R., Momtazee, C., 2017. Pregnancy: effect on multiple sclerosis, treatment considerations, and breastfeeding. Neurotherapeutics 14 (4), 974-984.

Nelson, L.M., Franklin, G.M., Jones, M.C., 1988. Risk of multiple sclerosis exacerbation during pregnancy and breast-feeding. JAMA 259, 3441-3443.

Confavreux, C., Hutchinson, M., Hours, M.M., Cortinovis-Tourniaire, P., Moreau, T., 1998. Rate of pregnancy-related relapse in multiple sclerosis. Pregnancy in multiple sclerosis group. N. Eng.1 J. Med. 339, 285-291.

Poser, S., Poser, W., 1983. Multiple sclerosis and gestation. Neurology 33, 1422-1427.

Sadovnick, A.D., Eisen, K., Hashimoto, S.A., et al., 1994. Pregnancy and multiple sclerosis. A prospective study. Arch. Neurol. 51, 1120-1124.

Mueller, B.A., Zhang, J., Critchlow, C.W., 2002. Birth out comes and need for hospitalization after delivery among women with multiple sclerosis. Am. J. Obstet. Gynecol. 186, 446-452.

Van der Kop, M.L., Pearce, M.S., Dahlgren, L., et al., 2011. Neonatal and delivery outcomes in women with multiple sclerosis. Ann. Neurol. 70, 41-50.

Finkelsztejn, A., Fragoso, Y.D., Ferreira, M.L., et al., 2011. The Brazilian database on pregnancy in multiple sclerosis. Clin. Neurol. Neurosurg. 113, 277-280.

Kelly, V.M., Nelson, L.M., Chakravarty, E.F., 2009. Obstetric outcomes in women with multiple sclerosis and epilepsy. Neurology 73, 1831-1836.

Chen, Y.H., Lin, H.L., Lin, H.C., 2009. Does multiple sclerosis increase risk of adverse pregnancy outcomes? A population-based study. Mult. Scler. 15, 606.

Dahl, J., Myhr, K.M., Daltveit, A.K., Hoff, J.M., Gilhus, N.E., 2005. Pregnancy, delivery, and birth outcome in women with multiple sclerosis. Neurology 65, 1961-1963.

Dahl, J., Myhr, K.M., Daltveit, A.K., Gilhus, N.E., 2008. Pregnancy, delivery and birth outcome in different stages of maternal multiple sclerosis. J. Neurol. 255, 623-627.

Hellwig, K., Brune, N., Haghikia, A., et al., 2008. Reproductive counseling, treatment and course of pregnancy in 73 German MS patients. Acta. Neurol. Scand. 118, 24-28.

Jalkanen, A., Alanen, A., Airas, L., 2010. Pregnancy outcome in women with multiple sclerosis: results from a prospective nationwide study in Finland. Mult. Scler. 16, 950-955.

Ramagopalan, S., Yee, I., Byrnes, J., et al., 2012. Term pregnancies and the clinical characteristics of multiple sclerosis: a population based study. J. Neurol. Neurosurg. Psychiatry 83, 793-795.

Thompson, D.S., Nelson, L.M., Burns, A., Burks, J.S., Franklin, G.M., 1986. The effects of pregnancy in multiple sclerosis: a retrospective study. Neurology 36, 1097-1099.

Weinshenker, B.G., Hader, W., Carriere, W., Baskerville, J., Ebers, G.C., 1989. The influence of pregnancy on disability from multiple sclerosis: a population-based study in Middlesex County, Ontario. Neurology 36, 1438-1440.

Jokubaitis, V.G., Spelman, T., Kalincik, T., et al., 2016. Predictors of long-term disability accrual in relapse-onset multiple sclerosis. Ann. Neurol. 80 (1), 89-100.

Runmarker, B., Andersen, O., 1995. Pregnancy is associated with a lower risk of onset and a better prognosis in multiple sclerosis. Brain 118 (Pt 1), 253-261.

D'Hooghe, M.B., Haentjens, P., Nagels, G., D'Hooghe, T., De Keyser, J., 2012. Menarche, oral contraceptives, pregnancy and progression of disability in relapsing onset and progressive onset multiple sclerosis. J. Neurol. 259, 855-861.

D'Hooghe, M.B., Nagels, G., Uitdehaag, B.M., 2010. Long-term effects of childbirth in MS J. Neurol. Neurosurg. Psychiatry 81, 38-41.

Portaccio, E., Ghezzi, A., Hakiki, B., et al., 2011. Breastfeeding is not related to postpartum relapses in multiple sclerosis. Neurology 77, 145-150.

Langer-Gould, A., Huang, S.M., Gupta, R., et al., 2009. Exclusive breastfeeding and the risk of postpartum relapses in women with multiple sclerosis. Arch. Neurol. 66, 958-963.

Gulick, E.E., 2002. Influence of infant feeding method on postpartum relapse of mothers with MS. Int. J. MS Care 4, 4-14.

Fragoso, Y.D., Boggild, M., Macias-Islas, M.A., et al., 2013. The effects of long-term exposure to disease-modifying drugs during pregnancy in multiple sclerosis. Clin. Neurol. Neurosurg. 115 (2), 154-159.

Hughes, S.E., Spelman, T., Gray, O.M., et al., 2014. Predictors and dynamics of postpartum relapses in women with multiple sclerosis. Mult. Scler. 20, 739-746.

Alroughani, R., Alowayesh, M.S., Almed, S.F., et al., 2018. Relapse occurrence in women with multiple sclerosis during pregnancy in the new treatment era. Neurology 90 (10), e840-e846 Mar 6.

Jesus-Ribeiro, J., Correia, I., Martins, A.I., et al., 2017. Pregnancy in multiple sclerosis: a Portuguese cohort study. Mult. Scler. Relat. Disord. 17, 63-68.

Polman, C.H., Reingold, S.C., Banwell, B., et al., 2011. Diagnostic criteria for multiple sclerosis: 2010 revisions to the McDonald criteria. Ann. Neurol. 69 (2), 292-302.

Hellwig, K., Haghikia, A., Rockhoff, M., Gold, R., 2012. Multiple sclerosis and pregnancy: experience from a nationwide database in Germany. Ther. Adv. Neurol. Disord. 5 (5), 247-253.

Lu, E., Wang, B.W., Guimond, C., Synnes, A., Sadovnick, D., Tremlett, H., 2012. Diseasemodifying drugs for multiple sclerosis in pregnancy: a systematic review. Neurology 79 (11), 1130-1135.

Fernández, O, Delvecchio, M., Edan, G., et al., 2018. Survey of diagnostic and treatment practices for multiple sclerosis (MS) in Europe. Part 2: progressive MS, paediatric MS, pregnancy and general management. Eur. J. Neurol. 25 (5), 739-746.

Ayres-De-Campos, D., Cruz, J., Medeiros-Borges, C., Costa-Santos, C., Vicente, L., 2015. Lowered national cesarean section rates after a concerted action. Acta. Obstet. Gynecol. Scand. 94 (4), 391-398.

Rabei, N.H., El-Helaly, A.M., Farag, A.H., El-Naggar, A.K., Etman, M.K., El-Moteily, M.M., 2017. Intrapartum fetal head circumference and estimated fetal weight as predictors of operativedelivery. Int. J. Gynaecol. Obstet. 137 (1), 34-39. 